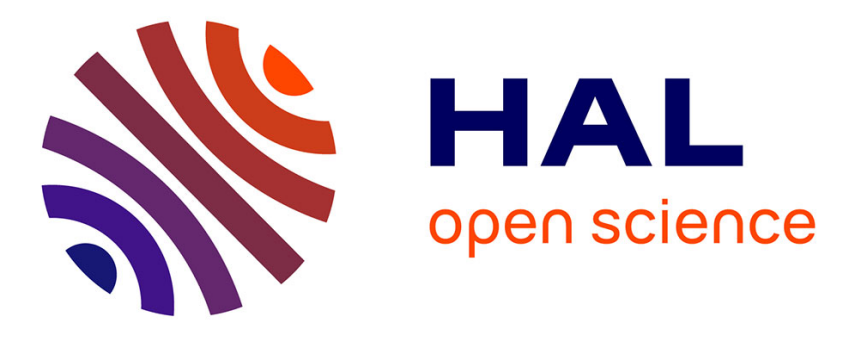

\title{
Using Road IDs to Enhance Clustering in Vehicular Ad hoc Networks
}

Mohamed Hadded, Paul Mühlethaler, Rachid Zagrouba, Anis Laouiti, Leila Azouz Saidane

\section{- To cite this version:}

Mohamed Hadded, Paul Mühlethaler, Rachid Zagrouba, Anis Laouiti, Leila Azouz Saidane. Using Road IDs to Enhance Clustering in Vehicular Ad hoc Networks. IWCMC' 2015. International Wireless Communications \& Mobile Computing Conference, Aug 2015, Dubrovnik, Croatia. hal-01211450

\section{HAL Id: hal-01211450 \\ https://hal.science/hal-01211450}

Submitted on 6 Oct 2015

HAL is a multi-disciplinary open access archive for the deposit and dissemination of scientific research documents, whether they are published or not. The documents may come from teaching and research institutions in France or abroad, or from public or private research centers.
L'archive ouverte pluridisciplinaire HAL, est destinée au dépôt et à la diffusion de documents scientifiques de niveau recherche, publiés ou non, émanant des établissements d'enseignement et de recherche français ou étrangers, des laboratoires publics ou privés. 


\title{
Using Road IDs to Enhance Clustering in Vehicular Ad hoc Networks
}

\author{
Mohamed Hadded ${ }^{* \ddagger}$, Paul Muhlethaler ${ }^{\dagger}$, Rachid Zagrouba ${ }^{\ddagger}$, Anis Laouiti ${ }^{*}$ and Leila Azouz Saidane $\ddagger$ \\ *TELECOM SudParis, CNRS Samovar, UMR 5157, France \\ ${ }^{\dagger}$ INRIA, BP 105. 78153 Le Chesnay Cedex, Paris-Rocquencourt, France

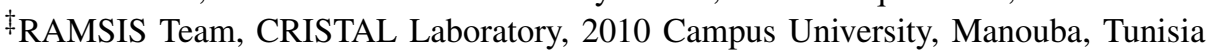 \\ \{mohamed.hadded, anis.laouiti\}@it-sudparis.eu, paul.muhlethaler@inria.fr, rachid.zagrouba@cristal.rnu.tn, \\ leila.saidane@ensi.rnu.tn
}

\begin{abstract}
Vehicular ad hoc networks (VANETs) where vehicles act as mobile nodes is an instance of Mobile Ad hoc NETworks (MANETs), which are essentially developed for intelligent transportation systems. A challenging problem when designing communication protocols in VANETs is coping with high vehicle mobility, which causes frequent changes in the network topology and leads to frequent breaks in communication. The clustering technique is being developed to reduce the impact of mobility between neighboring vehicles. In this paper, we propose an Adaptive Weighted Cluster Protocol for VANETs, which is a road map dependent and uses road IDs and movement direction in order to make the clusters structure as stable as possible. The experimental results reveal that AWCP outperforms four other most commonly used clustering protocols in terms of control packet overhead, the packet delivery ratio, and the average cluster lifetime, which are the most usual metrics used for comparing performance.
\end{abstract}

Keywords-VANET, MANET, Clustering, GPS, MAP, Road ID.

\section{INTRODUCTION}

Research on Vehicular Ad hoc Networks (VANETs) has attracted increasing interest over recent years due to its capability to improve road safety by using Vehicle To Vehicle (V2V) and/or Vehicle To Infrastructure (V2I) communications [1]. It can also be used to improve traffic management conditions and to provide on-board infotainment such as Internet access, video streaming, etc. VANETs are characterized by the self-organization of the nodes, where their nodes can be vehicles, roadside units or sensors. Due to the varying vehicular densities caused by high vehicle mobility, supporting network connection requires a high communication overhead for exchanging and updating topology information. For instance, in a fully distributed VANET, each vehicle is required to maintain its own connectivity to its one hop neighboring vehicles. Without using expensive components such as central points, establishing a hierarchical clustering structure within the network can reduce the relative mobility between neighboring vehicles, thereby reducing communication overhead [2]. Clustering allows the formation of organized groups and is used to coordinate channel access, simplify routing, and security. In VANETs, each vehicle is equipped with a digital road map and a positioning system, e.g. Garmin Nuvi 50 GPS that allows it to obtain the time, its speed and position [3] and the ID of the road on which it is traveling. Therefore a clustering protocol for VANET network can be designed using a MAP digital and GPS based approach. However, the main challenge for clustering protocols in VANETs is ensuring topology stability by taking into account many mobility parameters. This should be performed with a minimum overhead. In this paper, we propose a multi-metrics based Adaptive Weighted Clustering Protocol (AWCP) that takes advantage of the geographic information of vehicles. The main contributions of the paper are listed below:

- We identify certain essential features that the clustering protocols must satisfy to build stable clusters in VANETs.

- We propose a Map and GPS based clustering protocol using the WCA algorithm [4] for VANET in which a vehicle only considers neighbors moving on the same road and in the same direction. To the best of our knowledge, this is the first attempt to design a clustering protocol by taking advantage ID of the road on which the vehicles are traveling.

- We evaluate the proposed protocol on realistic VANET mobility scenarios taken from the metropolitan area of San Jose (California) and we validate its performance by comparing it with other clustering protocols in the literature.

The rest of the paper is organized as follows. In Section 2, we present some relevant guidelines (in form of rules) to establish stable clusters in VANETs and a summary of related work. Section 3 describes our clustering protocol, called AWCP. Section 4 presents the simulation results and the performance evaluation. Finally, conclusions and future work are reported in Section 5.

\section{CONTEXT AND Related WORK}

\section{A. Clustering in VANET}

When a vehicle node wishes to participate in a cluster head election, it firstly collects all the necessary one-hop neighbors information. In this section, we identify the rules that the clustering protocol must satisfy in VANET networks with the aim to form stable clusters, where re-clustering is reduced, and cluster members lifetimes are prolonged.

Rule 1. The cluster head vehicles should have sufficiently powerful radios to be able to communicate with the members of their clusters. This implies that the cluster heads should be 
close to the center of the cluster. Thus, the vehicle that has the minimum average Euclidean distance to their direct neighbors can be elected to act as the cluster head. The average Euclidean distance is:

$$
\delta(i, t)=\frac{\sum_{j} \sqrt{\left(x_{i}-x_{j}\right)^{2}+\left(y_{i}-y_{j}\right)^{2}}}{n(i, t)}
$$

$n(i, t)$ is the number of vehicles connected directly to $i$ at instant $t$, where $j$ is any vehicle that is connected to $i$.

Rule 2. To form a stable cluster, the cluster head should have similar mobility characteristics as the vehicles within its cluster. Indeed, if cluster heads are elected without taking speed into account, the number of vehicles that will quickly move out of communication range from their cluster head will increase. Thus, the vehicle whose current speed is the closet to the mean value is elected as the cluster head. The average value of speed is:

$$
\rho(i, t)=\frac{\sum_{j} \nu(j, t)}{n(i, t)}
$$

$\nu(t, j)$ is the speed of vehicle $j$ at instant $t$, where $j$ is any vehicle that is in communication range of the cluster head candidate $i$.

Rule 3. The formation of a high number of clusters increases the overhead and the inter-cluster interference and degrades the network performance. To overcome this, the cluster mechanism should group all vehicles in the network with a small number of cluster heads. Thus, we restrict the vehicle that has the maximum number of neighboring vehicles should be elected to act as the cluster head. However, this number $i$ should be bounded by a capacity value $\beta_{i}$ which represents the maximum number of neighbors that vehicle $i$ can optimally handle as a cluster head.

Rule 4. To provide a stable cluster structure, the cluster members should always remain within the transmission range of their cluster heads. Thus, the clustering protocol should take into consideration the mobility features of VANETs (e.g. multiple roads including road junctions, opposite-direction of the traffic flow).

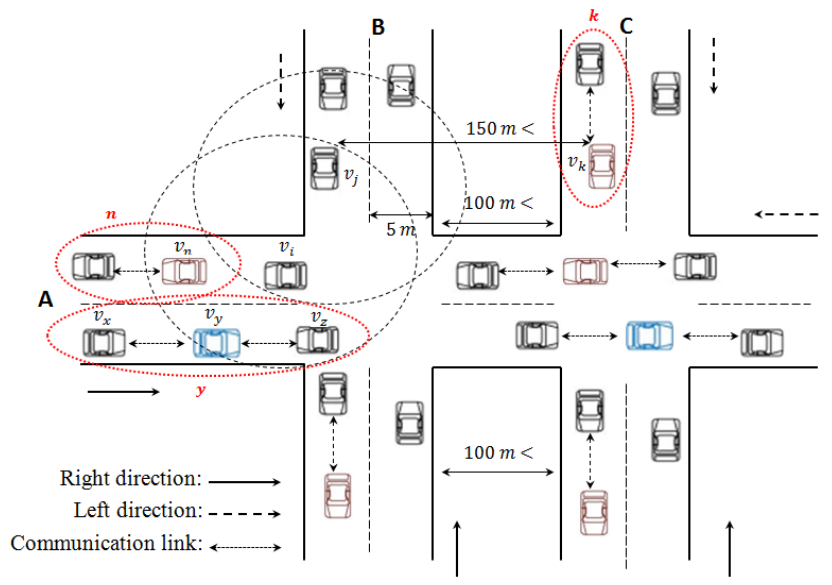

Fig. 1. Road-based clustering

Rule 4.1. The Mobility Direction (MD) is necessary information that can be used to form stable clusters. Indeed, the vehicles that are moving in different directions cannot remain within transmission range for a long period of time. Let us consider the VANET scenario shown in Figure 1. Vehicle $v_{i}$ can choose to join one of the two clusters $y$ or $n$ which are managed by the two vehicles $v_{y}$ and $v_{n}$, respectively. If vehicle $v_{i}$ joins cluster $y$, it will quickly leave the cluster and it will need to choose another cluster head. However, if vehicle $v_{i}$ joins cluster $n$, they will both be moving in the left hand direction and as a result it will remain a member of this cluster for a longer period of time.

Rule 4.2. The mobility direction is not always sufficient to insure clustering stability in VANETs. As shown in Figure 1, based on the mobility direction metric the vehicle $v_{j}$ can join cluster $k$ managed by the vehicle $v_{k}$ because they are moving on the same direction. However, they are not moving on the same road and vehicle $v_{k}$ may need to change its status and choose a new cluster head if the distance between the two roads becomes greater than the communication range of the cluster head. Moreover, based on the definitions of the mobility directions given in [5], the two vehicles $v_{i}$ and $v_{j}$ are considered to be moving in the same "left" direction and thus these vehicles can be grouped together to form a cluster. Since the two vehicles are not moving on the same road, vehicle $v_{i}$ will be out of the cluster $j$ after a short period of time and it will need to join a new cluster. Thus, the Road ID (RID) is critical information to provide a more stable cluster structure and to reduce the average number of times a vehicle must change clusters. In this paper, we impose that each vehicle only considers neighboring vehicles that are moving on the same road and in the same direction, and ignores control messages from vehicles on a different road and moving in the opposite direction.

\section{B. Related work}

Clustering is the best known method in VANETs to enable efficient resource allocation with low overhead and to reduce the relative mobility between neighboring vehicles. Several VANET research studies in the literature have focused on developing clustering protocols, most of which are based on MANET clustering techniques. However, none of the protocols proposed takes RID into account when forming clusters in VANETs. As a result, these protocols do not create stable clusters. Some of the most significant protocols are described below.

In [2], the authors proposed a multi-metric algorithm for cluster head elections, called Threshold-based Technique (TB), suitable for highway area. In addition to the position and the direction, this algorithm uses a speed difference metric as a new parameter to increase the cluster lifetime. The vehicles that are moving at high speed are regrouped into one cluster, while the vehicles moving at low speed are grouped into another cluster. An Adaptable Mobility-Aware Clustering Algorithm based on Destination positions, called AMACAD, is proposed and evaluated by Morales et al. [6]. The goal of this work has been to develop a clustering protocol with an efficient message exchange mechanism, which improves the clustering stability in VANETs. AMACAD performs clustering based upon information such as current location, vehicle velocity, relative destination and final destination of vehicles. A Multi-Head Clustering Algorithm, called Center-Position and 
Mobility (CPM), was proposed in [7]. This technique aims to create stable clusters and reduce re-clustering overhead by supporting single and multiple cluster heads. In the cluster head election phase, vehicles within communication are organized into clusters and one vehicle for each cluster is elected to act as a Master Cluster Head (MCH). Then, some cluster members in the cluster are selected to be Slave Cluster Heads (SCHs). In order to form stable clusters, the authors imposed that all the vehicles in a cluster are moving in the same direction.

Several other clustering algorithms designed for MANETs also work in VANETs and are frequently used for comparison with other VANET clustering protocols. For instance, the Lowest-ID clustering algorithm (LID) [8] is based on electing a node with the smallest ID as a cluster head. The HighestDegree algorithm (HD) [9] selects a node as a cluster head based on the nodes' connectivity. The node with the maximum number of neighbors becomes the cluster head. The Weighted Clustering Algorithm (WCA) [4] elects a node to act as a cluster head based on a combined weight which includes the number of its neighbors, their average distance and the node's average speed, and battery-life. MOBIC [10] is a Mobilitybased clustering algorithm designed for MANETs which is also used in VANETs. MOBIC is a mobility based version of the Lowest-ID algorithm and uses a signal power level metric to elect cluster heads. Table I compares the features of these protocols. It is clear from this table that while all of these protocols satisfy certain of these rules, none of them satisfies all of them. As result, these protocols may not work efficiently in VANET network. Thus, in this paper, we propose a clustering protocol, called AWCP, that uses road ID information in addition to the known parameters i.e., direction, connectivity, average speed. To the best of our knowledge, this is the first contribution to design a clustering protocol that uses road ID information and which satisfies all the above mentioned rules.

\section{AWCP DESCRIPTION}

\section{A. System Model}

Our algorithm is based on the assumption that each vehicle in a VANET can know its road ID via a digital road map and a positioning system, e.g. GPS (Global Positioning System) or a GALLILEO receiver that also allows it to obtain an accurate real-time three-dimensional geographic position (latitude, longitude and altitude), direction, speed and exact time.

TABLE I. Comparison of clustering protocols

\begin{tabular}{|c|c|c|c|c|c|c|c|}
\hline Protocols & WCA & HD & LID & CPM & AMACAD & TB & MOBIC \\
\hline Rule 1 & $\checkmark$ & & & $\checkmark$ & $\checkmark$ & & $\checkmark$ \\
\hline Rule 2 & $\checkmark$ & & & $\checkmark$ & $\checkmark$ & $\checkmark$ & \\
\hline Rule 3 & $\checkmark$ & $\checkmark$ & & & & & \\
\hline Rule 4.1 & & & & $\checkmark$ & $\checkmark$ & $\checkmark$ & \\
\hline Rule 4.2 & & & & & & & \\
\hline
\end{tabular}

\section{B. Cluster Head Election}

In this section, we present the cluster setup and maintenance mechanisms of AWCP in detail. Initially, all vehicles are in the Undecided State (US). To divide the network into clusters, each active vehicle changes its state to Cluster Head Candidate (CHC) and it starts to broadcast a HELLO message periodically containing all the necessary information $\langle V I D, R I D, M D$, position, speed $\rangle$ to its One-Hop neighbors $(\mathrm{OH})$. In order to form stable clusters, each vehicle uses RID and MD to filter out any vehicle that is moving on another road or in the opposite direction. Upon reception of a HELLO message from all its one-hop neighbors, each vehicle $i$ calculates its current weight $W(i, t)$ using the following function (3). The weight function consists of three parts, i.e., average-distance weight factor (1), average-speed weight factor (2), number of neighbors weight factor.

$W(i, t)=w_{1} * \delta(i, t)+w_{2} *|\nu(i, t)-\rho(i, t)|-w_{3} * n(i, t)$

Where $w_{1}, w_{2}$ and $w_{3}$ are the balancing factors such that $\sum_{k=1}^{3} w_{k}=1$. We note that the three weight factors are in conflict. For simplicity, we assume that all the factors should be minimized. In fact, the multiplication of the thirst weight factor by (-1) allows us to transform a maximization to minimization. Then, each node $i$ broadcasts a beacon message containing all the necessary information for the $\mathrm{CH}$ election algorithm $\langle V I D, R I D, M D, W, C H-I D\rangle$. Vehicle $i$ announces itself as a $\mathrm{CH}$ by assigning its own ID to the $\mathrm{CH}$-ID field of the election beacon. When a vehicle $i$ receives beacons from its one-hop neighbors, it sorts its neighbor list $O H_{i}$ according to the weights received in the beacons, and then it executes the cluster head election algorithm to change its status from $\mathrm{CH}$ to Cluster Member (CM), Cluster Gateway (CG) or remain $\mathrm{CH}$.

$$
W(i, t)=\left\{\min W(j, t) \forall j \in O H_{i}\right\}(4), \quad n(i, t) \leq \beta_{i}
$$

The vehicle $i$ that satisfies the two properties (4) and (5) at instant $t$ is elected as the $\mathrm{CH}$. Then, all vehicles that are within transmission range of the $\mathrm{CH}$ become $\mathrm{CMs}$ or CGs and are not allowed to participate in another cluster head election procedure. The $\mathrm{CH}$ election algorithm terminates once all the vehicles either become a $\mathrm{CH}, \mathrm{CM}$ or a $\mathrm{CG}$. Algorithm 1 outlines the details of the $\mathrm{CH}$ nodes' election. It is executed by each vehicle $i$ having at least one neighboring vehicle. In Algorithm 1, $i, j$, and $x$ represent three vehicles which are moving in the same road and on the same direction and are participating in the $\mathrm{CH}$ election process. In addition, ITJ_Interval is the time interval for a $\mathrm{CH}$ vehicle to broadcast the Invite-To-Join (ITJ) message, PRE_Interval is the time interval for a $\mathrm{CM}$ to signal its presence to its $\mathrm{CH}$, while CH_Timeout_Interval is the time interval for a vehicle to elect itself as a $\mathrm{CH}$, if it did not receive any ITJ messages during this period.

\section{Cluster maintenance}

In VANETs, a vehicle can join or leave a cluster at any time. These two operations will have only local effects on the topology of the cluster if the vehicle is a CM. However, if the vehicle is the $\mathrm{CH}$, it must hand over the responsibility to one of the very close cluster members before leaving the cluster. The first reason for that is to maintain the cluster structure even if the current $\mathrm{CH}$ leaves. The second reason is to avoid using the re-clustering algorithm and thus no re-clustering overhead is generated when the $\mathrm{CH}$ leaves the cluster. Then, the current 
$\mathrm{CH}$ will order the $\mathrm{CM}$ to switch to $\mathrm{CH}$ and switch its own state to CM. Each cluster head periodically broadcasts an ITJ messages to its one-hop neighbors. Once a US or CHC vehicle receives an ITJ message, and if it wishes to join the cluster, it will check the received signal strength. The US or CHC vehicle will consider the ITJ message to be valid if its signal strength is greater than the predefined threshold denoted by Pr_Threshold. When receiving a valid ITJ message, the vehicle sends a Request-To-Join (RTJ) message including the vehicle's ID, road ID and direction. When the $\mathrm{CH}$ receives the RTJ message, it checks the road ID on which the requesting vehicle moving and, if it is moving in the same direction, the $\mathrm{CH}$ sends an acknowledgment (ACK) including its ID number. After the reception of the ACK, the corresponding vehicle becomes a $\mathrm{CM}$ of this cluster. Once a US vehicle becomes a CM, it is not allowed to participate in another cluster head election procedure. Moreover, if a CM receives an ITJ message from another neighboring $\mathrm{CH}$ moving on the same road and in the same direction, the vehicle will switch from the CM state to the CG state. Figure 2 shows the vehicle state transitions diagram. A vehicle remains in the $\mathrm{CM}$ state as long as it receives an ITJ message from its $\mathrm{CH}$ every ITJ_Interval. When the CM vehicle does not receive an ITJ message from its $\mathrm{CH}$ during CH Timeout Interval, it considers that it has lost contact with the $\mathrm{CH}$ and thus switches its state to $\mathrm{CHC}$. Each $\mathrm{CH}$ updates a time stamp field for each $\mathrm{CM}$ based on the presence messages (PRE-MSG) received. The $\mathrm{CH}$ removes a $\mathrm{CM}$ from its cluster members list if the difference between the current time and the last time stamp of the PRE-MSG message received from it is greater than CM_Timeout_Interval. The $\mathrm{CH}$ will change its state to $\mathrm{CHC}$, if its list of cluster members is empty. Moreover, when two or three $\mathrm{CHs}$ moving on the same road and in the same direction receive an ITJ message from each other, only one of them will keep its $\mathrm{CH}$ responsibility based on the the weight function (3), while the others will switch to a CM.

\section{SIMULATION RESULTS AND PERFORMANCE EVALUATION}

This section presents NS2 simulation results to evaluate the performance of AWCP. The objectives of the evaluation are to: (1) Evaluate the performance of AWCP under realistic and different mobility scenarios, (2) Evaluate and compare the efficiency of AWCP with the most popular clustering protocols in the literature, (3) Test the efficiency of AWCP in reducing the number of states changed per vehicle.

\section{A. Simulation Scenarios}

We generated a realistic VANET environment by selecting a real highway area from a digital map which took into account road directions, road intersections, and traffic rules. Figure 3 shows a metropolitan area from the Map of San Jose (California) of size $4000 m \times 4000 m$ exported from OpenStreetMap (OSM) and edited using Java OpenStreetMap Editor (JOSM). Then MOVE and SUMO [11] were used respectively to generate vehicle traffic scenarios and to simulate the area with vehicular traffic. To generate vehicular traffic by MOVE and SUMO, we defined a vehicle flow which described a swarm of vehicles in each direction. The parameters of each vehicle flow consisted of the maximum number of vehicles, the starting road and destination of the flow, the time to start

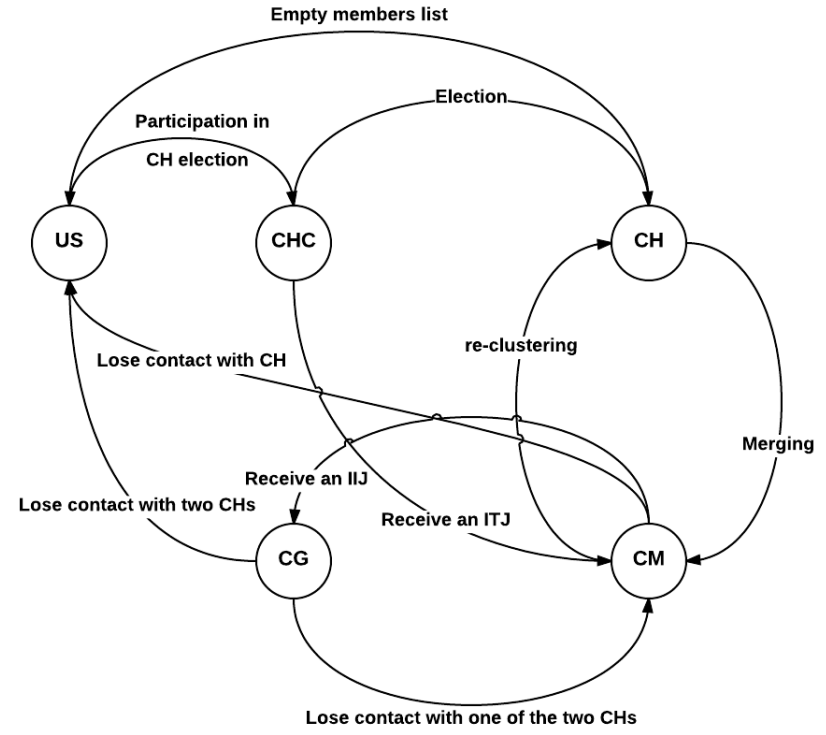

Fig. 2. Vehicle state transition diagram

and end the flow and the probabilities of turning in different directions at each junction ( 0.4 to go straight, 0.3 to turn left and 0.3 to turn right). Then the traffic traces generated by MOVE were used in the ns 2.34 simulations. All the tests were performed on different VANET scenarios taking into account different vehicle densities and data loads: Low, Medium, High and Very High. The features of the VANET scenarios and the simulation parameters used in our experiments are summarized in Tables II and III, respectively. AWCP is evaluated based on
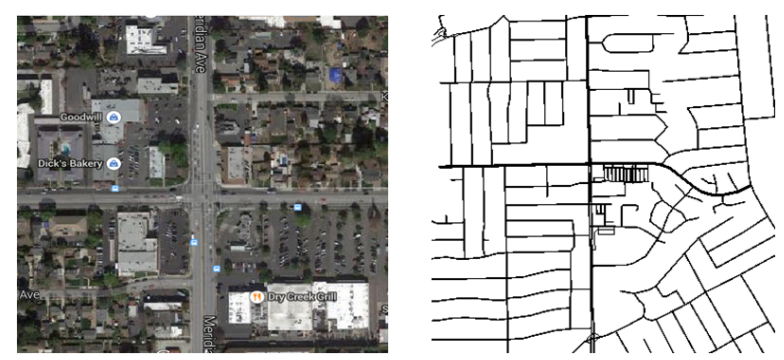

Fig. 3. San Jose (California) urban area captured from Google Maps (left) and exported to a VANET network topology by using MOVE/SUMO (right)

TABLE II. VANETs scenarios

\begin{tabular}{ccc}
\hline Scenario & Number of vehicles & Number of CBR sources \\
\hline Low $($ S1) & 25 & 5 \\
Medium $($ S2) & 50 & 15 \\
High $($ S3) & 100 & 25 \\
Very High $($ S4) & 150 & 35 \\
\hline
\end{tabular}

three of the most widely used Quality of Service (QoS) metrics in this domain [2]: The Average Cluster Lifetime (ACL), which is the average time period from the moment when a vehicle becomes a $\mathrm{CH}, \mathrm{CM}$ or $\mathrm{CG}$ to the time when it changes its state. The Control Packet Overhead (CPO), which is the rate of AWCP control packets used to form and maintain the cluster structures. Finally, the Packet Delivery Ratio (PDR), which is the ratio of the number of data packets that are correctly delivered to their destinations. 
TABLE III. Simulation parameters in ns-2

\begin{tabular}{cc}
\hline Parameter & Value/Protocol \\
\hline Simulation time & $100 \mathrm{~s}$ \\
Vehicle speed & $120-150 \mathrm{~km} / \mathrm{h}$ \\
Propagation model & Two Ray Ground \\
Medium Capacity & $6 \mathrm{Mbps}$ \\
PHY/MAC Layer & $I E E E 802.11 p$ \\
Transmission range & $1000 \mathrm{~m}$ \\
Transport Layer & $U D P$ \\
CBR Packet Size & 512 bytes \\
CBR Data Rate & $0.1 \mathrm{Mbps}$ \\
\hline
\end{tabular}

\section{B. AWCP parameters}

We have used the min-EUDT AWCP configuration found by NSGA-II in [12] which is the most balanced setting of parameters, that optimizes the AWCP QoS metrics. Table I shows the parameters of AWCP and their values. These parameters are four timers, four counters and three weighting factors.

TABLE IV. AWCP PARAMETERS

\begin{tabular}{ccc}
\hline Parameter & Type & Value \\
\hline Hello_Interval & $\mathbb{R}$ & $0.78 \mathrm{~s}$ \\
Election_Interval & $\mathbb{R}$ & $0.16 \mathrm{~s}$ \\
ITJ_Interval & $\mathbb{R}$ & $7.23 \mathrm{~s}$ \\
PRE_Interval & $\mathbb{R}$ & $9.16 \mathrm{~s}$ \\
CH_Timeout_Interval & $\mathbb{R}$ & $12.75 \mathrm{~s}$ \\
CM_Timeout_Interval & $\mathbb{R}$ & $12.7 \mathrm{~s}$ \\
Cluster_Size & $\mathbb{Z}$ & 50 \\
Pr_Threshold & $\mathbb{R}$ & $7.23 \mathrm{E}-16 \mathrm{w}$ \\
Distance_Weight_factor $\left(w_{1}\right)$ & $\mathbb{R}$ & 0.716 \\
Speed_Weight_factor $\left(w_{2}\right)$ & $\mathbb{R}$ & 0.204 \\
Neig_Weight_factor $\left(w_{3}\right)$ & $\mathbb{R}$ & 0.07 \\
\hline
\end{tabular}

\section{AWCP performance evaluations}

In this section, we evaluate and compare the performance of AWCP with other well known clustering protocols proposed in the literature, namely WCA, LID, HD and CPM. Figure 4 shows the ACL of all the algorithms for different VANET scenarios. This figure shows that the ACL is increased by respectively $63.6 \%, 62.2 \%, 59 \%$ and $45.5 \%$ on average when AWCP is used compared to the WCA, HD, LID, and CPM protocols. Therefore, we can conclude that the protocol proposed provides stable clusters which have a long lifetime. As AWCP takes into account road IDs and movement directions to form clusters, the CMs will be associated with their $\mathrm{CHs}$ for a longer period of time. We can also note that CPM performs better than WCA, LID and HD, because the CPM protocol forms clusters based on the mobility direction.

The Clustering Protocol Overhead (CPO) of AWCP and the other protocols for various of VANETs scenarios is shown in Figure 5. It is clear from this figure that our protocol has a lower overhead than the other protocols. In fact, AWCP reduces the $\mathrm{CPO}$ by respectively $38.8 \%, 37.2 \%, 37.1 \%$ and $47.2 \%$ on average compared to WCA, HD, LID and CPM. There are two reasons why AWCP decreases the overhead. Firstly because the maximization of the cluster heads' duration and the cluster members' duration decreases the number of control messages required to elect new cluster heads and to join a new cluster, respectively. Secondly, the minimization of the number of the clusters reduces the amount of ITJ messages broadcasted by the cluster heads.

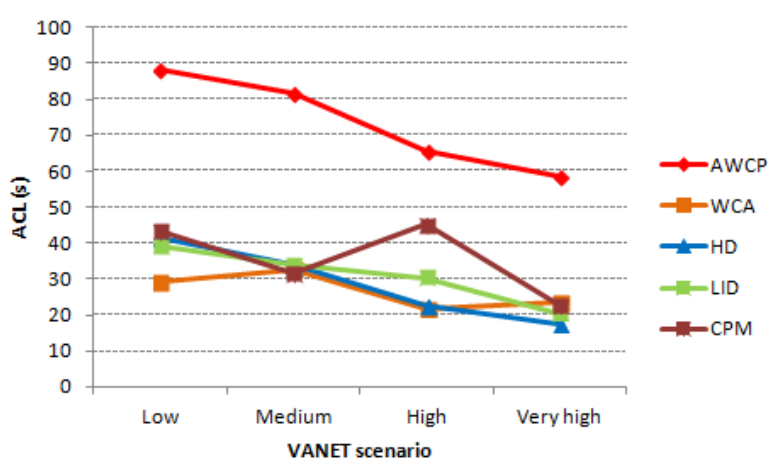

Fig. 4. ACL results for different VANET scenarios

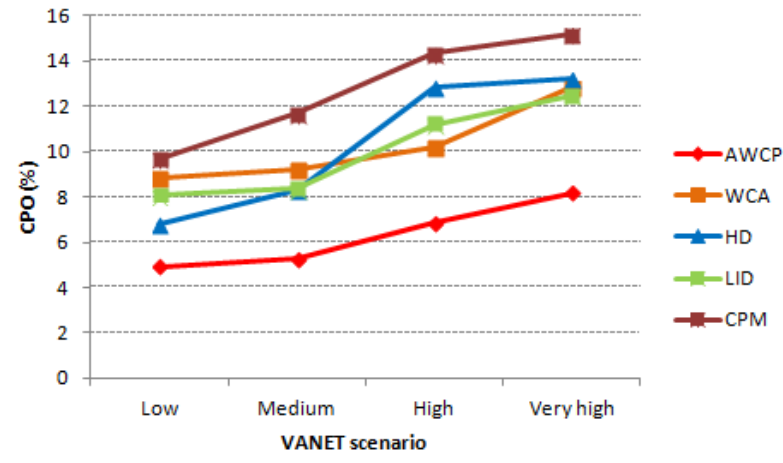

Fig. 5. CPO results for different VANET scenarios

Figure 6 shows the Packet Delivery Ratio (PDR) achieved by each clustering protocol for various VANET scenarios. It clearly shows that for varying traffic densities the AWCP protocol gives the best performance in terms of PDR, except for the High scenario, where WCA, HD and LID deliver a higher data rate. Although the network performance in terms of throughput significantly decreases when the vehicle density increases, on average, AWCP guarantees a better PDR than the other protocols. This is due to the fact that AWCP does not generate an excessive clustering overhead and thus the data packets are transmitted to their destination vehicles with a lower collision rate.

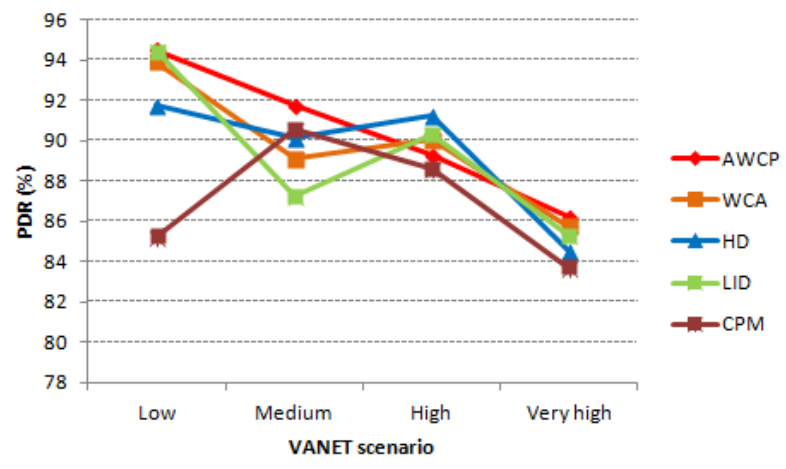

Fig. 6. PDR results for different VANET scenarios

Figure 7 shows the average US duration (the average duration in which the vehicles are in the US state) with respect to 
road traffic density. We note that the AWCP protocol provides a smaller US average duration than the other protocols, except for Low and High scenarios where CPM and ACWP behave similarly. Moreover, it can be seen from this figure that the average US duration increases significantly for HD, WCA and LID in the high scenario, while it still remains reasonable for both the AWCP and CPM protocols. Figures 8 and 9 show

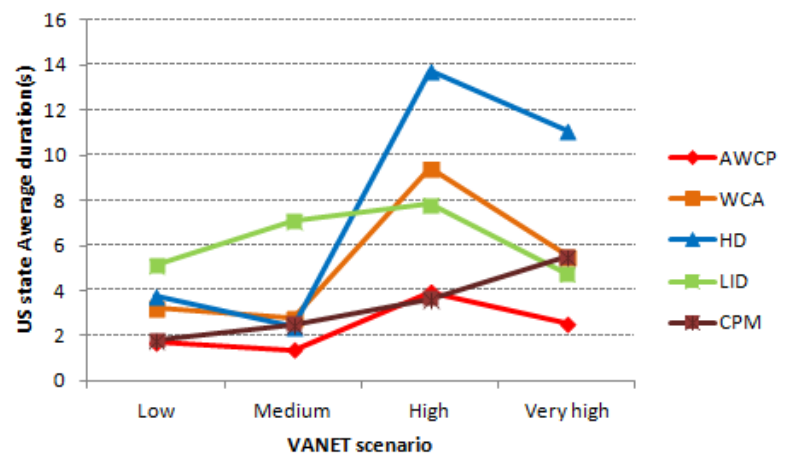

Fig. 7. The average duration in which the vehicles are in the US state for different VANET scenarios

the number of changes of states for each vehicle during the simulation time for the S3 and S4 scenarios. In both figures 8 and 9, we note that AWCP causes the lowest number of transitions. For instance, vehicle 14 in Figure 8 kept its sate throughout the simulation time when AWCP was used, while it changes its state 3, 4, 5 and 8 times when CPM, WCA, LID and $\mathrm{HD}$ were used, respectively. These results can be explained by the fact that AWCP avoids the problem of merging multiple clusters into a single cluster at road junctions.

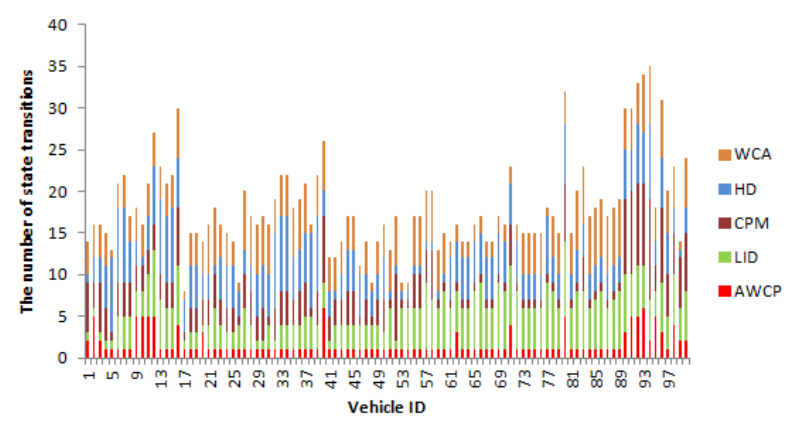

Fig. 8. The number of vehicles' state transitions for the S3 VANET scenario

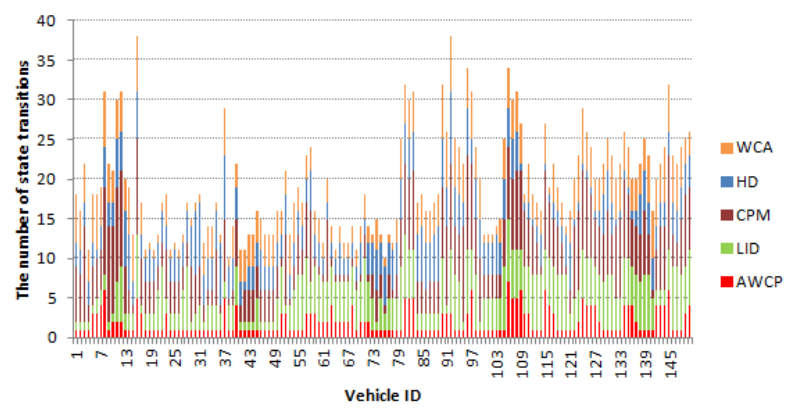

Fig. 9. The number of vehicles' state transitions for the S4 VANET scenario

\section{CONCLUSion}

Designing of efficient clustering protocols is an important issue in VANETs due to the rapid changes in network topology and the lack of infrastructure. In this paper, we identify the essential properties that clustering protocols must satisfy to form stable clusters in VANETs, and we propose an optimized clustering protocol, called AWCP, whose objective is to maximize the lifetime of the cluster heads and cluster members. AWCP is a MAP and GPS based approach which takes advantage of knowing the road ID and the direction in which the vehicles are traveling. Our method of selecting cluster heads based on mobility features and a weight function is the key to achieving a more stable cluster. The experimental analysis shows that AWCP clearly improves the clustering performance in VANETs in terms of cluster lifetime duration and communication overhead compared to the well known clustering protocols WCA, LID, HD. This is also the case when AWCP is compared with another protocol CPM, which considers both the vehicles position and the direction of movement.

The ns 2 simulation results presented in this paper are very encouraging and justify continuing in this research direction. We plan to design a cross-layer architecture (MAC/AWCP) to improve channel access efficiency in VANETs in which each cluster head is responsible for assigning bandwidth to all the members of its cluster.

\section{REFERENCES}

[1] Q. Tse, "Improving message reception in vanets," in MobiSys, Krakow, Poland, Jun. 2009

[2] Z. Y. Rawashdeh and S. M. Mahmud, "A novel algorithm to form stable clusters in vehicular ad hoc networks on highways," EURASIP Journal on Wireless Communications and Networking, Jan. 2012.

[3] J. D. Carswell and T. Tezuka, "A modular neural network approach to improve map-matched gps positioning," in W2GIS, China, Dec. 2006, pp. 76-89.

[4] M. Chatterjee, S. K. Das, and D. Turgut, "A weighted clustering algorithm for mobile ad hoc networks," Cluster Computing, vol. 5, no. 2, pp. 193-204, Apr. 2002.

[5] H. A. Omar, W. Zhuang, and L. Li, "Vemac: A tdma-based mac protocol for reliable broadcast in vanets," IEEE Transactions on Mobile Computing, vol. 12, no. 9, pp. 1724-1736, Sep. 2013.

[6] M. M. C. Morales, C. S. Hong, and Y. C. Bang, "An adaptable mobilityaware clusteringalgorithm in vehicular networks," in APNOMS, Taipei, Taiwan, Sep. 2011, pp. 1-6.

[7] S. C. Lo, Y. J. Lin, and J. S. Gao, "A multi-head clustering algorithm in vehicular ad hoc networks," International Journal of Computer Theory and Engineering, vol. 5, no. 2, pp. 242-247, Apr. 2013.

[8] M. Gerla and J. C. Tsai, "Multicluster, mobile, multimedia radio network," Wireless Networks, vol. 1, no. 3, pp. 255-265, 1995.

[9] A. Ramalingam, S. Subramani, and K. Perumalsamy, "Associativity based cluster formation and cluster management in ad hoc networks," in IEEE HiPC, Bangalore, India, Dec. 2002, pp. 1-6.

[10] P. Basu, N. Khan, and T. Littl, "A mobility based metric for clustering in mobile ad hoc networks," in ICDCS Workshop, Arizona, Apr. 2001, pp. $413-418$.

[11] F. Karnadi, Z. Mo, and K. chan Lan, "Rapid generation of realistic mobility models for vanet," in IEEE WCNC, Hong Kong, China, Mar. 2007, pp. 2506-2511.

[12] M. Hadded, R. Zagrouba, A. Laouiti, P. Muhlethaler, and L. A. Saidanel, "A multi-objective genetic algorithm-based adaptive weighted clustering protocol in vanet," in IEEE CEC, Sendai, Japan, May 2015. 